\section{Contact dermatitis related to constituent of an orthopaedic wool}

Tests were carried out to determine the cause of an acute vesicular eczema that occurred in six patients with plaster-of-Paris casts lined with orthopaedic wool. The reaction was concluded to have been due to one of the constituents of the wool.

\section{Patients, methods, and results}

Six patients, aged between 7 and 40, presented with an acute vesicular eczema coinciding with the application of a Gypsona plaster-of-Paris cast lined with Softexe orthopaedic wool. The time from application of the cast to presentation varied from four days to three weeks. To determine whether
Failure to recognise contact allergy after prolonged occlusive contact may lead to secondary infected eczema, cellulitis, or even infected fractures, though no major complication has yet been seen. The itching, pain, and sweating resulting from contact dermatitis beneath a constricted cast or bandage may occur at any time from a few days to several weeks after application. The symptoms and signs are easy to confuse with those due to constriction or friction caused by the cast. Simply splitting or replacing the cast will not be sufficient treatment. If the diagnosis is suspected the entire cast and lining bandage should be removed, subject to the necessary treatment for the fracture, when different splinting materials should be used. Exposure is usually sufficient for treating the rash, though a mild topical steroid may be necessary.

Contact allergy, though rare, may be avoided by eliminating allergens from cast materials. The manufacturers have told us that a new formulation of Softexe is being prepared without Clestol.

Results of patch tests at 48 and 96 hours

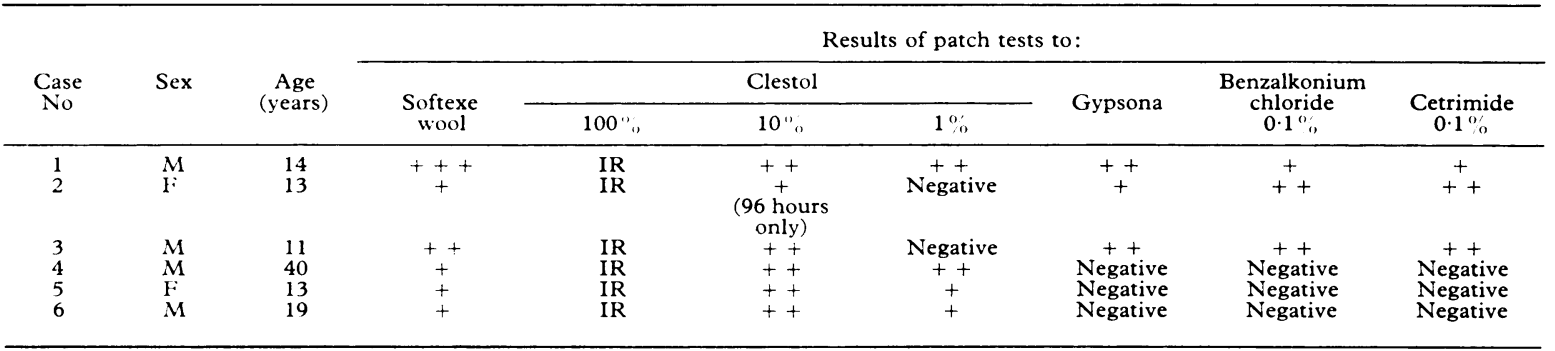

$\mathrm{IR}=$ Irritant reaction

the eczema was an allergic reaction samples of four chemicals used in the production of Softexe were obtained from the manufacturers (Cuxson, Gerrard and Co Ltd). Patch tests were applied using Finn chambers under Scanpor. Each ingredient was applied neat and also in $10 \%$ and $1 \%$ dilutions in yellow soft paraffin. Additional patch tests were performed on each patient to the European standard battery and to Gypsona, benzalkonium chloride, cetrimide, and Softexe wool. They were read 48 and 96 hours after application.

All six patients developed allergic reactions to Softexe, while three were also allergic to Gypsona, benzalkonium chloride, and cetrimide (table). Of the Softexe ingredients tested, only one (sodium dioctyl sulphosuccinate, also known as Clestol) produced an allergic reaction; this occurred in all six patients. Four patients developed an allergic reaction to a $1 \%$ solution of Clestol. Neat Clestol also produced an irritant reaction in all cases. None of the three other chemicals produced allergic reactions.

Eight normal subjects and 10 with non-inflammatory skin disease were patch tested to neat Clestol and to $10 \%$ and $1 \%$ dilutions of this substance. Neat Clestol produced an irritant reaction in 12 of the 18 subjects; the $10 \%$ and $1 \%$ dilutions caused neither irritant nor allergic reactions.

\section{Comment}

Softexe is a fairly new lining bandage used beneath plaster casts and is usually placed in direct contact with the skin. Its properties permit comfortable, firm application of various self-setting cast materials. Among its constituents, which include wetting agents and an acrylic binder, is an anionic surfactant called Clestol (sodium dioctyl sulphosuccinate). This is a known contact allergen. ${ }^{1}$ Contact allergy to benzalkonium chloride in Gypsona has been described. ${ }^{2}$ Three of the patients in our series were also allergic to this substance. Cross-sensitisation has been observed between benzalkonium chloride and other quaternary ammonium compounds-for example, cetrimide. ${ }^{3}$ Sodium dioctyl sulphosuccinate is chemically unrelated, and cross-sensitisation seems unlikely. Three patients (cases 1, 2, and 3) exhibited positive patch-test reactions to benzalkonium chloride and cetrimide as well as to sodium dioctyl sulphosuccinate. Repeat testing to Softexe and its constituents in appropriate dilutions over six months later confirmed this pattern of allergy. It is unlikely that they exhibited the "angry back syndrome." The other three patients, however, developed positive patch tests to Softexe and Clestol alone. In addition, the tests on normal controls suggest that the affected patients had a true allergy.
We are grateful to Messrs Cuxson, Gerrard and Co (Dressings) Ltd for their co-operation and to Mrs Janet Owen for help in preparing this paper.

${ }^{1}$ Fisher AA. Contact dermatitis. 3rd ed. Philadelphia: Lee and Febinger, 1976.

2 Staniforth P. Allergy to benzalkonium chloride in plaster of Paris after sensitisation to cetrimide; a case report. F Bone foint Surg $[B r] 1980$; 62:500-1.

${ }^{3}$ Lovell CR, Staniforth P. Contact allergy to benzalkonium chloride in plaster of Paris. (In press.)

(Accepted 5 August 1981)

Bristol Royal Infirmary, Bristol BS2 8HW

P STANIFORTH, MB, FRCS, senior orthopaedic registrar

C R LOVELL, MB, MRCP, senior registrar in dermatology (present address: St John's Hospital for Diseases of the Skin, London WC2H 7BJ)

\section{Captopril-associated lymphadenopathy}

Captopril, a converting-enzyme inhibitor, is now being used increasingly to treat arterial hypertension and congestive cardiac failure. ${ }^{1}$ Severe side effects of the drug, such as proteinuria ${ }^{2}$ and agranulocytosis, $^{3}$ have been reported. We report two cases of reversible lymphadenopathy in patients treated with captopril.

\section{Case reports}

Case 1-A 37-year-old woman had had untreated hypertension for five months. She had had no previous important illnesses and, apart from her raised blood pressure, physical examination and laboratory tests showed nothing abnormal. After six weeks of treatment with captopril $300 \mathrm{mg}$ daily as the only antihypertensive agent she was admitted to hospital with painful lymph nodes in both groins, the left axilla, and the neck. The liver and spleen were not palpable. Leucocyte count was $4.6 \times 10^{9} / 1$, with $25 \%$ 
eosinophils. Erythrocyte sedimentation rate was $3 \mathrm{~mm}$ in the first hour, and immunoglobulins, antinuclear antibodies, and complement titres were normal. Liver enzyme activities were also normal. Microscopic examination of the enlarged lymph nodes showed unspecified adenitis. A chest $x$-ray film was normal. Captopril was immediately withdrawn, and within two weeks the lymph nodes were not palpable and the eosinophil count was normal.

Case 2-A 47-year-old man had had primary hypertension for five years. $\mathrm{He}$ had no known liver disease, and liver enzyme activities were normal before he received captopril. His blood pressure became normal with $150 \mathrm{mg}$ of captopril daily and for three months all laboratory tests were normal. $\mathrm{He}$ was subsequently admitted with painful palpable lymph nodes in both groins, the left axilla, and the back of the right knee. His temperature was $38.3^{\circ} \mathrm{C}$. The leucocyte count was $6 \times 10^{9} / 1$, with $5 \%$ eosinophils. Erythrocyte sedimentation rate was $6 \mathrm{~mm}$ in the first hour. Serum aspartate and alanine transferase activities were slightly raised $(0.90 \mu \mathrm{kat} / 1$, normal $<0.75)$. Captopril was immediately withdrawn. After one week the lymph nodes were not palpable and all laboratory tests were normal.

\section{Comment}

The lack of any previous disease of the lymphatic system in these two patients and the rapid recovery within a few days of withdrawal of the drug suggest a drug-related lymphadenopathy. Though the true nature of this lymphadenopathy is not clear, the occurrence of enlarged lymph nodes associated with administration of captopril merits attention.

${ }^{1}$ Atkinson AB, Robertson JIS. Captopril in the treatment of clinical hypertension and cardiac failure. Lancet 1979; ii :836-9.

2 Case DB, Atlas SA, Mouradian JA, Fishman RA, Sherman RL, Laragh JH. Proteinuria during long-term captopril therapy. $\mathcal{F} A M A \quad 1980 ; 244$ : 346-9.

${ }^{3}$ van Brummelen P, Willemze R, Tan WD, Thompson J. Captoprilassociated agranulocytosis. Lancet $1980 ; \mathrm{i}: 150$.

(Accepted 5 August 1981)

Department of Medicine, University Hospital, S-75014 Uppsala, Sweden

$\mathrm{H} \AA$ ÅERG, MD, associate professor

C MÖRLIN, MD, registrar

Department of Medicine, Central Hospital, S-63188 Eskilstuna, Sweden

G FRITHZ, MD, head of cardiology unit

\section{Long-term morbidity of herpes zoster ophthalmicus}

Ocular complications occur in half the patients with herpes zoster ophthalmicus, ${ }^{1}$ and, though the prognosis has improved considerably with the use of corticosteroids, antibiotics, and artificial tear preparations, ${ }^{2}{ }^{3}$ the disorder remains potentially serious, sometimes resulting in blindness. This study reports the long-term morbidity of herpes zoster ophthalmicus in 13 patients still requiring treatment 12 months to 23 years after the onset of the disease.

\section{Patients, methods, and results}

Patients were included in the study if they were attending an ophthalmology clinic for the treatment of complications one year or more after an attack of herpes zoster ophthalmicus. All but one were known to have received the standard recommended form of treatment for herpes zoster ophthalmicus ${ }^{4}$ early in the disease: an intensive course of topical antibiotics and corticosteroids was given during admission to hospital for about one week and antiglaucoma treatment and artificial tear preparations were provided if required. Low-dose topical steroids were subsequently continued for several months on an outpatient basis. Information about the initial treatment in case 13 was not available.

Assessment of each patient included measurement of visual acuity, intraocular pressure, and tear production, as well as ophthalmoscopy and examination with a slit-lamp biomicroscope.

A progressive reduction in visual acuity was found in 11 of the 13 patients (table); in five patients vision was reduced to finger counting, hand movements, or perception of light, and one patient (case 11) had his eye excised. The major complication, found in nine patients, was loss of corneal sensation associated with recurrent keratitis and scarring of the cornea. The eyelids of five of these patients (cases $6,8,10,11$, and 12) required suturing (tarsorrhaphy) to protect their anaesthetic corneas. Despite tarsorrhaphy the neuroparalytic keratitis in two of these patients resulted in spontaneous perforation of the eye. One of these, a 40-year-old woman (case 8) with systemic lupus erythematosus requiring treatment with systemic steroids, developed perforation of the cornea 20 months after the attack of herpes zoster ophthalmicus. By the time of this survey she had received three corneal grafts and undergone a cataract extraction and an operation for glaucoma. The second patient, an 88 -year-old man (case 11), developed a corneal abscess and spontaneous perforation that necessitated excision of the eye 22 months after the onset of herpes zoster ophthalmicus. Chronic glaucoma and recurrent uveitis were found in seven patients; in one of them (case 13) these complications were still requiring treatment 23 years after the onset of herpes zoster ophthalmicus. In three of these patients (cases $8,9,13$ ) associated cataracts resulted in severe visual impairment. Scarring of the eyelids and eyelash abnormalities (trichiasis) were not major complications except in one patient (case 2), who required four plastic surgery procedures for cicatricial ectropion of the upper lid. Three patients (cases $4,5,8$ ) complained of persistent postherpetic neuralgia.

\section{Comment}

Spontaneous perforation of an anaesthetic cornea has been described as a frequent complication of herpes zoster ophthalmicus, often requiring excision of the eye. ${ }^{1}$ Since the introduction of modern therapeutic methods perforation of the eye has become a rare complication: in a series of over 500 patients presenting with herpes zoster ophthalmicus only one subsequently sustained perforation of an eye and this responded satisfactorily to medical treatment. ${ }^{3}$ In the present study perforation of the eye occurred in two patients, one of them elderly and the other immunologically compromised. Both patients required extensive surgical intervention, the older patient having the eye excised. The late occurrence of corneal perforation, nearly two years after the onset of herpes zoster ophthalmicus, suggests that patients in these categories should be carefully followed up for a long time. Glaucoma and recurrent uveitis may similarly require treatment for an extended period after the initial episode of herpes zoster ophthalmicus.

I thank the consultant ophthalmologists at Coventry and Warwickshire Hospital for permission to report on their patients, and Dr Heather $M$ Beaumont for help in preparing the manuscript.

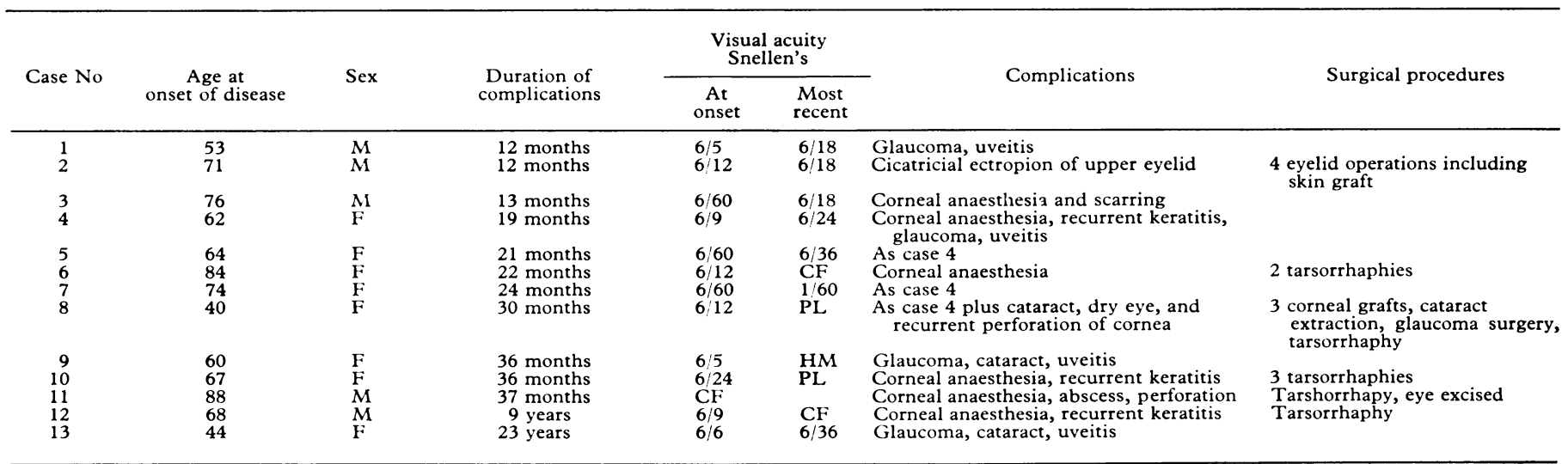

